\title{
A HIGH EFFICIENY LAMB WAVE LENS FOR SUBSURFACE IMAGING
}

\author{
A. Atalar, H. Köymen ${ }^{\dagger}$ \\ Bilkent University, Ankara TLRKEY \\ $\dagger$ Middle East Technical University, Ankara, TURIEY
}

\begin{abstract}
A conventional scanning acoustic microscope lens excites all the possible modes of acoustic waves in the solid structure under examination. The excited leaky modes contributes significantly to the high contrast obtained in the images. But since all such modes exist simultaneously, the interpretation of the images is not straightforward. We propose a new lens geometry which can be used with acoustic microscopes to image layered solid structures. This new lens can focus the acoustic waves in only one of the Lamb wave modes of the layered solid with a ligh efficiency. The images obtained are easy to interpret and the subsurface sensitivity is high.
\end{abstract}

\section{INTRODUCTION}

Leaky layer waves such as Rayleigh waves or generalized Lamb waves can be excited in a planar layered structure immersed in a liquid by bulk waves incident at the surface at some critical angles 11]. The critical angles are determined mainly by layer thickness, elastic properties of the layered structure and the frequency of operation. When a scanning acoustic microscope is used to image a laycred solid structure, almost all possible acoustic wave modes are excited in the structure, because the acoustic microscope lens creates all incidence angles at the object interface. Some of these excited modes are bulk waves, and some are leaky modes that eventually return to the liquid medium. The efficiency of excitation is rather low, because an appreciable part of input power is wasted at angles where there is no subsurface excitation. An interference of specularly reflected rays with leaky modes gives rise to the well-known $V(Z)$ effect which is responsible for the high contrast in the acoustic images. From such images one hopes to detect flaws like delaminations, layers under stress, changes in elastic parameters, etc. Unfortunately, the presence of all modes simultaneously makes the inages difficult to interpret. Although there exists methods of recovering elastic parameters of the material under test from the received signal, the results of these computations are not very accurate and most of the time the inversion problem is ill-conditioned. In this paper we propose a different lens design that creates images easy to interpret. The lens can be realized eitler in reflective or refractive modes. The reflective design makes use of a planar ring bulk-wave transducer and a section of a full cone as the reflecting surface. The refrac- tive design, on the other hand, resembles a conventional acoustic microscope lens except that it employs a conical focusing surface rather than a spherical one. Both designs can excite subsurface acoustic waves with a high efficiency. Images obtained with sucl a lens are presented.

\section{EXCITATION OF LEAKY MODES}

A layered solid supports acoustic wave modes referred to as Rayleigh-like (LR) waves [2] and generalized Lamb waves in adclition to the bulk waves. The LR wave is like a Raylcigh wave and it is confined to the surface. This wave is dispersive, and the dispersion is determined by the wavelength and the thickness of the layer(s). The generalized Lamb wave modes are like Lamb wave modes in a plate and they are also dispersive.

When the layered solid is immersed in a liquid, all these modes become leaky, as their energy is radiated into the liquid medium. In this case, it is also possible to excite these modes by a bulk wave insonification in the liquid medium [3]. Corresponding to each mode there exists a critical angle of incidence [1]. Since the modes are dispersive, the critical angles depend on the frequency. The dispersion curves can be obtained by examining the reflection coefficient at the liquid-layered solid boundary [5]. At the critical angles the reflection coefficient has a phase transition.

In this paper, we consider solids containing only one laver. We calculated the dispersion characteristics for a number of structures using the reflection coefficient [6]. Fig. 1 shows the dispersion curves of llayleigh-like wave and the Lamb wave modes for a copper layer on an aluminum substrate. The horizontal axis is the wavenumber-thickness product, $k_{t s} d$. Ilere, $k_{i s}$ is the shear wavenumber of the substrate and $d$ is the tlichness of the layer. As opposed to the common convention, the vertical axis is the critical angle of incidence. The curves are obtained numerically by calculating the reflection cocficicut at the licpuicl-layered solid interface. For small $k_{t s} d$ values only the LR mode exists. For $k_{t s} d=0$, the critical angle, $\theta$, is equal to the Rayleigh critical angle of the substrate. As $k$ ts $d$ increases, the critical angle changes and approaclies to the Rayleigl critical angle of the layer material. The first Lamb wave mode (M2) is excited only when $k_{t s} d$ is greater than 3.66. For greater $k_{t s} l$ values, the critical angle varies toward the shear critical angle of the layer material. Iligher order Lamb wave modes (MI3, M., etc.) appear at even greater values of $k_{t s} d$. At the cut-off point, the critical angle is equal to the shear critical angle of the substrate material. 


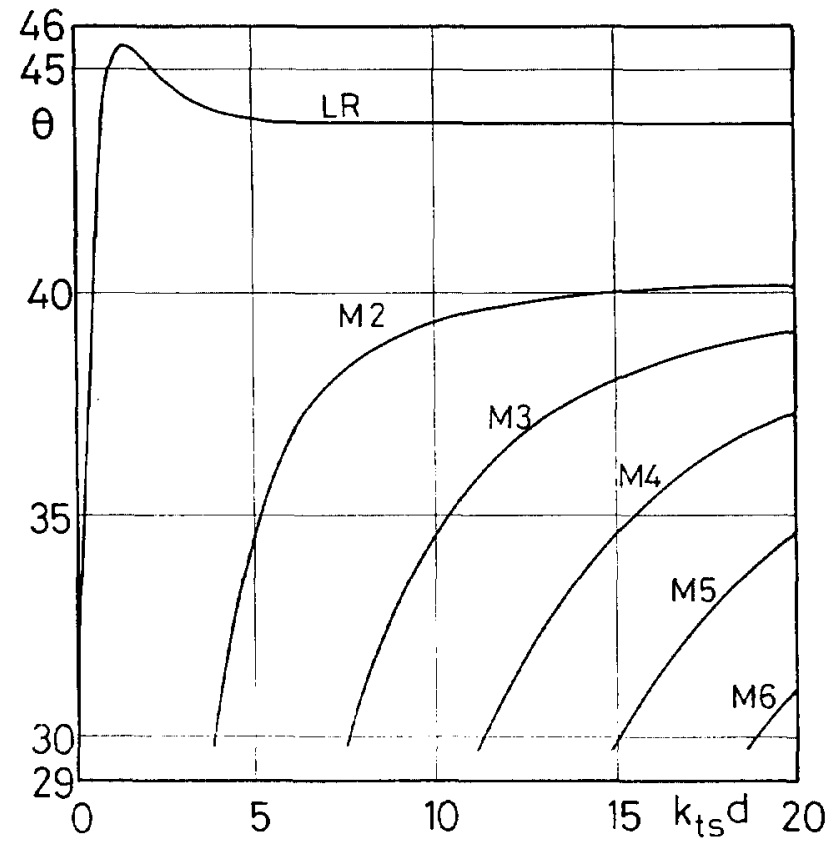

Figure 1: Excitation angle of various modes from the liçuid side as a function of $k_{t s} d$ for a copper layer on an aluminum substrate

Our aim is to be able to excite these modes efficiently from the liquid side using a wedge transducer [7,8]. An efficiency measure of the wedge transducer for a particular mode can be deduced from the variation of the reflection coefficient phase at the critical angle. In particular, the slope of the phase variation is an important parameter [9]. This slope is proportional to the Schoch displacement, $\Delta_{S}$. For optimum excitation efficiency, the wedge width, $W$, must be selected as [10] $W=0.62 \Delta_{S} \cos \theta$, where $\theta$ is the critical angle. In Fig. 2 we plot $\Delta_{s} / \lambda$ with respect to $k_{t s} d$ for the same solid structure where $\lambda$ is the wavelength in the liquid. For LR mode, $\Delta_{S} / \lambda$ is the lowest, while the same value for the Lamb wave modes exhibit a minimum for $k_{t s} d$ values very close to their respective cut-off values. For a practical werlge width, very large $\Delta_{S} / \lambda$ values must be avoided. Therefore, for efficient Lamb wave excitation, the minimum points of the curves in Fig. 2 must be targeted. In our particular example, a wedge angle slightly greater than $30^{\circ}$ is suitable.

\section{LAMB WAVE LENS}

In the previous section we have determined the conditions for efficiently launching the Lamb wave modes in a layered structure by a wedge transducer. A wedge transducer is not suitable to use in an imaging system due to its poor resolution ability. A method of focusing Lamb waves was described earlier [11]. That system utilized a cylindrical reflecting surface and a wedge translucer. Ilowever, its resolution was not very good because of the small convergence angle. Ilere, we describe a new configuration with a better resolution. The basic idea is to use a full conical wave rather than a section of it. The creation of a conical wave can be achieved either by a conical transducer $[12,13\}$ ar by reflection or refraction from a suitable conical surface. The geometry of

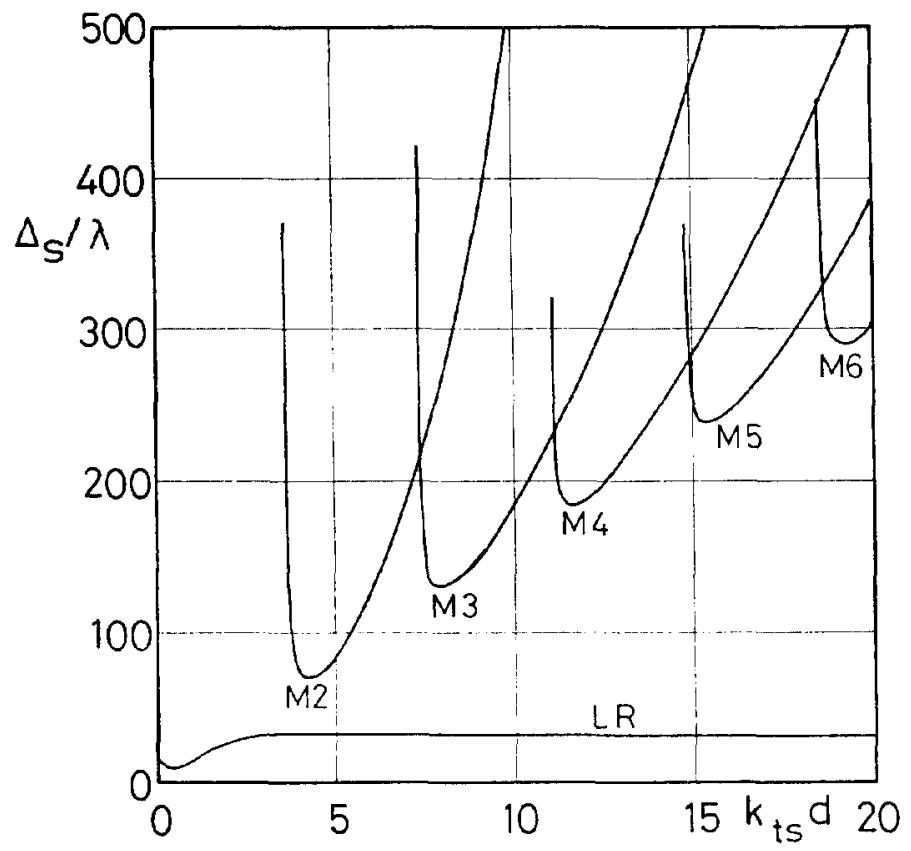

Figure 2: Schoch displacement of various modes in wavelength units as a function of $k_{t s} d$ for a copper layer on an aluminum substrate

the proposed reflecting Lamb wave lens is illustrated in Fig. 3. It consists of a transducer and a refecting surface placed right next to it. The transducer has a ring shape and it is positioned parallel to the surface under examination. The reflecting surface is metallic and it has a conical shape. The conical surface is situated below the transducer in such a way that the axis of the cone is normal and concentric with the ring transducer. The acoustic waves produced by the transducer will first hit the conical reflecting surface before they reach the object surface. It can be easily proved that all the refected rays from the conical surface are incident at the object surface at the same angle. The incidence angle is equal to the twice the inclination of the conical surface with respect to its axis. If this incidence angle is chosen to be a critical angle for Lamb waves, almost all the bulk wave energy will be converted to a leaky Lamb wave. The excited evanescent Lamb waves converge at the point where the axis of the cone intersects the object surface. At this point, these waves constructively add and form a focus. In the absence of any inhomogeneity at the focus, the waves will diverge and leak back into the liquid medium. Upon refection from the conical surface at the symmetrical side, they will be detected by the transducer and induce an electrical signal. If an inhomogeneity is present at the focus point, the received electrical signal will be disturbed and will diminish in amplitude. IIence, this focusing wedge assembly will act like a Lamb wave lens. To avoid detection of specularly reflected rays, the distance between the object surface and the lens must be sufficiently small. This requirement is satisfied if we set the distance between the transducer and the olject surface, $Z$, as $Z<R / \tan \theta$, where $R$ is the outer radius of the ring transducer. To have a clearance between the conical mirror and the object, the inner radius of the transhucer, $r$, must satisfy $r>R-Z \tan (\theta / 2)$. 


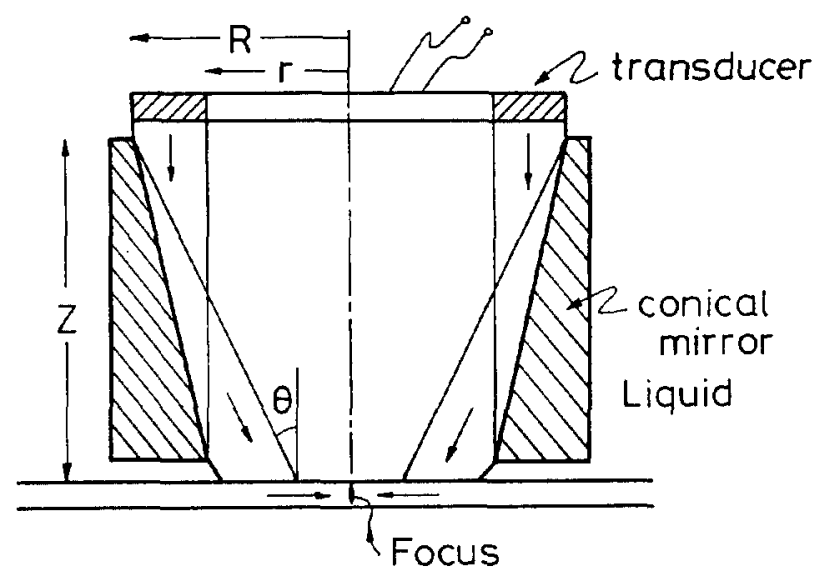

Figure 3: Geometry of the reflective type Lamb wave lens

One apparent problem of the lens geometry is that the incidence angle of rays at the object surface is fixed and this angle may not coincide with a critical angle for Lamb waves for another layer thickness. This problem can be solved easily by adjusting the excitation frequency. Since the Lamb wave modes are dispersive, the critical angle of a Lamb wave mode can be made equal to the fixed angle of the Lamb wave lens at the proper frequency. The experimental determination of frequency is very easy: one needs to tune the frequency until the maximum signal is received.

An imaging system using such a lens will have an axial resolution equal to the thickness of the layer, since the Lamb wave modes exist predominantly in the layer. On the other hand, the lateral resolution is not easy to define. There will be a perturbation in the received signal if a flaw lies anywhere within the circularly converging leaky wave, although the disturbance will be greatest when the flaw is right at the focus. The point response function will be determined by the wavelength of the excited Lamb wave mode as well as the size of the lens opening. For very small defects the second effect may be ignored. In this case, since the $f$-number of the resulting lens is very small and no aberrations are involved, it is possible to obtain lateral resolutions better than a wavelength.

Since the bandwidth of most transducers are not very wide, for some samples it may be impossible to hit a critical angle within the tuning range. Therefore, one needs a series of Lamb wave lenses with different cone angles to be able to cover all the possible samples.

The same focusing effect can be realized by a conical refracting surface as delineated in Fig. 4. In fact, this configuration is preferable because of its simblarity to an acoustic microscope lens. The spherical recess of the conventional lens is replaced by a conical one. An antireflection layer must be coated on the conical surface to reduce mismatch loss. The central part has to be blocked by absorbing materials to avoid acoustic waves that may come out of this region. The inclination of the refracting surface can be determined easily from the Snell's Law. If a ligh velocity material like sapphire is used as the buffer rod medium, the inclination of the conical refracting surface, $\gamma$, will be alnost

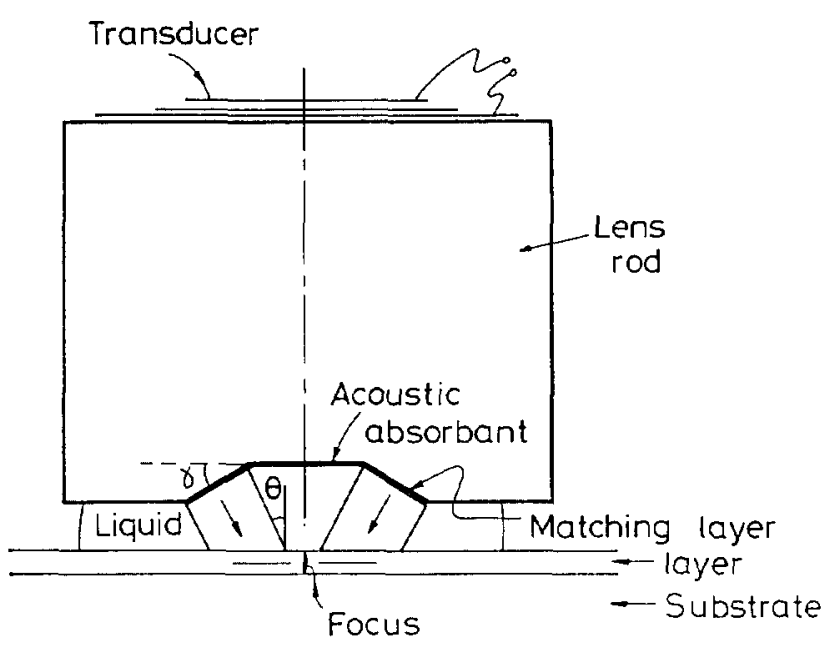

Figure 4: Geometry of the refractive type Lamb wave lens

equal to the critical angle of the object.

\section{IMAGING USING FOCUSED LAMB WAVES}

To illustrate the subsurface imaging ability of the Lamb wave lens, we fabricated a reflective type lens working around $5.5 \mathrm{MIIz}$ generating a conical wave at $31^{\circ}$ incidence angle. The fabricated lens covers a $180^{\circ}$ angular range rather than the full $360^{\circ}$ range. So, it is only one half of the geometry shown in Fig. 3. In this case, if there is no defect or inlomogeneity at the focus point, there will be no received energy. Therefore, this geometry forms a zero-background lens. The disadvantage of this configuration is the difficulty of frequency acljustment. Fig. 5 shows an image obtained with this lens at $5.6 \mathrm{MHz}$. At this frequency the wavelength of Lamb waves is about $0.52 \mathrm{~mm}$. The object is a polished surface of a $1 \mathrm{~mm}$ thick copper shect epoxy-bonded to an aluminum substrate $\left(k_{t s} d=11.5\right)$. Fig. 1 reveals that $\mathrm{N} \cdot 4$ node of Lamb waves is excited. There are $0.6,1.0$, and $1.5 \mathrm{~mm}$ deep indentations on the bonded surface of aluminum. The image does not display these subsurface indentations on the aluminum surface, but it depicts lots of other structure which may be attributable to the defects in the copper hyer or in the epoxy bond between the copper and the aluminum. Obviously, none of these defects are visible from the top surface. Considering the wavelength, the resolution of the image is very good. In lig. 6 we present the images oblained for the same region when the freguency is clanged to $5.0 \mathrm{MJIz}\left(k_{t s} l=10.3\right)$ and $6.4 \mathrm{MIIz}$ $\left(k_{t s} d=13.2\right)$. Fig. 1 indicates that no efficient Lamb wave excitation is possible at these frequencies. Comparison of the images with Fig. 5 reveals that for both images the resolution and the contrast are highly degraded. We must point out that in the upper image some features are visible at the positions corresponding to the subsurface holes. The same features are probably also in Fig. 5, but burried under the high contrast features. Therefore, the correct frequency adjustment is essential to obtain good images. The frequency (and the incidence angle) must be suitably 


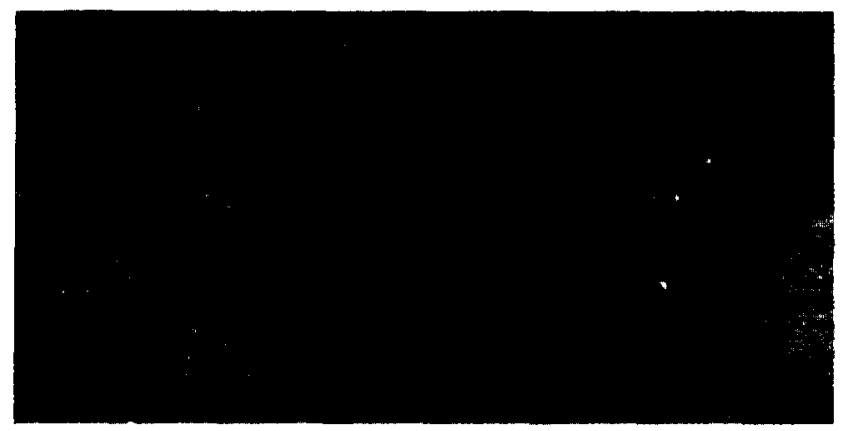

Figure 5: Iaml wave image of a $1 \mathrm{~mm}$ thick copper layer and an aluminum substrate at $5.6 \mathrm{MIIz}$. The vertical dimension of the image is $25 \mathrm{~mm}$.

chosen to meet one of the curves of Fig. 1.

\section{CONCLUSIONS}

The Lamb wave lens can complement the conventional lens in acoustic microscopes for some applications due to its inherent ability to focus waves in a subsurface laver with a high efficiency. The new lens is suited to image layered struct ures with little latcral vatiation. To use such a lens, the acoustic microscope must lave the ability to vary its operation frequency. The frequency must be tuned to excite a suitalile Lamb wave mocle. This new lens does not have a critical focal plane as the spherical lenses lave. The only requirement is to kecp the distance to the object below a certain limit. Although the variations in this distance may affect the signal output, it is not very critical. I[ence, in a mechanically scanned imaging system, the mechanical accuracy requirement can be relaxed.

The refracting mode lens is completely compatible with the existing manufacturing technology of the conventional acoustic lenses. Because of the simplicity of manufacturing a conical surface, it is plausible to build Lamb wave lenses with diameters in the order of $10 \mathrm{~km}$. For such small lenses the path that must be traveled in the Jighly lossy liquid medium is quite small, enalbling the focusing system to work at very high frequencies, possibly at frequencies not achicrable with the conventional acoustic microscope lens.

\section{References}

[1] C.W. Farnell, and C.K. Jen, "Excitation of acoustic beams in layered substrates," Rocue Phys. Appl, vol. 20, pp. 305310.1985

[2] G.W. Farnell and E.L. Adler, "Elastic Wave propagation in thin laycrs," in Pliysical Acoustics, Eds. W.P. Mason and R.N. Thurston, (Academic) vol. IX, pp. 35-127, 1972.

[3] D.B. Bogy, and S.M. Ciracewsk, "On the plane-wave reflection coefficient and nonspecular reflection of bounded beams for hall-spaces," J. Acoust. Soc. Am., vol. 7.t, pp. 591-599. 1983.
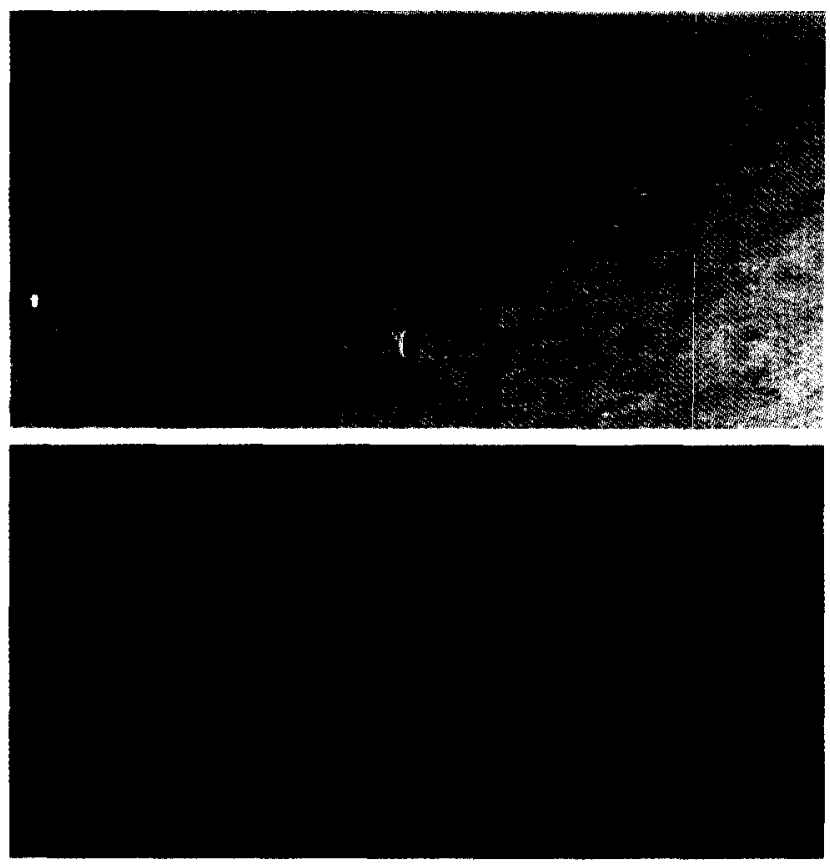

Figure 6: Images of the same sample at $5.0 \mathrm{MIIz}$ (upper) and at $6.4 \mathrm{MHI}$ (lower).

[4] T. Kundu, "On the nonspecular reflection of bounded acoustic beams," J. Acoust. Soc. Am, vol 83, pp. 18-2.1, $198 \mathrm{~s}$.

[5] J. Wolf, T.D.K. Ngoc, R. Kille, and W.G. Mayer, "Investigation of Lamb waves having a negative group velocity," J. Acoust. Soc. Am., vol. 83, pp. 122-126, 1988.

[6] D.L. Folds and C.D. Loggins, "Transmission and reflection of ultrasonic waves in layered media," J. Acoust. Soc. Am. vol. 62, pp. 1102-1109, 197 T.

[7] Il.L. Bertoni and T. Tamir, "Claracteristics of werlge transducers for acoustic surface waves", IEEE Truns Sonics $t /$ trason., vol. 22, pp. $415-420,1975$.

[8] J. Fraser, P.T. Khuri-lakub and G.S. Kino. "The resign of eflicient broadband wedge transelucers," Appl. Phys. Ioll. vol. 32, pp. 698-700, $197 \mathrm{~s}$.

[9] A.II. Nayfeh, and D.E. Chimenti, "Reflection of finite acoustje beams from loaded and stiffened half-sparce," J. Acoust. Srac. Am., vol. 75, pp. 1360-1368, 198.1

[10] A. Atalar and II. Körmen, "Use of a conical axicon as a surface acoustic wave focusing device", IEEE Truns. Cllmuson. Ferro. and Fieq. Cont, vol. 34, pp. 53-63, 1957.

[11] A. Atalar, ll. Köymen, O. Yomisgiler, "Measurement of sensitivity of different wave modes 10 subsurface defects." in

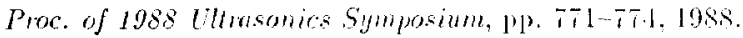

[12] R.L. Bacr, B.T. Khuri-Yakub, and C.S. Küno, "Conical transducer for generation of acoustic waves in fluids," Appl. Phys. Lelt., vol. 42. pp. 573-57.1, 1983.

[13] S. Ayter, "Focusing surface wayes using conical transducers," in Proc of 1987 IEEE thirason. Symp. pp. 301 301. 1987. 